\title{
Interactions between dendritic cells, probiotic bacteria and the adipokine leptin
}

\author{
S. C. Knight ${ }^{1}$, N. R. English ${ }^{1}$ and A. I. F. Blakemore ${ }^{2}$ \\ ${ }^{1}$ Antigen Presentation Research Group, Imperial College London, Northwick Park \& St Mark's Campus, Watford Road, \\ Harrow HA1 3UJ, UK and ${ }^{2}$ Genomic Medicine Group, Imperial College London, Hammersmith Campus,
}

Du Cane Road, London W12 ONN, UK

Perinodal adipose tissue (PAT) has specialised properties; functional dendritic antigen-presenting cells (DC) are abundant in PAT but rare in adipose tissues distant from nodes ${ }^{(1)}$, and in human subjects, as previously reported in rodents, PAT normally has tissue-specific properties, being high in PUFA ${ }^{(2)}$. Several properties of PAT are altered in the inflammatory bowel disease (IBD) Crohn's disease. It has been shown first that DC in PAT lose MHC Class II expression and capacity to stimulate T-cells ${ }^{(1)}$ and second that site-specific changes in fatty acids are lost or altered in Crohn's disease ${ }^{(2)}$. Finally, hypertrophy of adipose tissue and increases in adipokines including leptin occur in Crohn's disease ${ }^{(2)}$.

DC are found between gut epithelial cells with veils protruding into the lumen making direct contact with commensal bacteria. Gut DC can then migrate via afferent lymphatics, entering lymph nodes via a perinodal sinus immediately below and connected with PAT. Potential anti-inflammatory benefits of probiotic bacteria include the capacity to affect the function of DC. Thus, bacterial components can decrease production of IL-12 and promote production of IL-10 in $\mathrm{DC}^{(3)}$. By contrast, leptin, in addition to its widely-accepted role in energy homeostasis and reproduction, acts as a pro-inflammatory cytokine promoting development of DC. In the present study, therefore, effects of probiotic bacteria on the potential of DC to respond to stimulation with leptin have been investigated by examining their expression of leptin receptors.

There are multiple leptin receptor splice forms, including a trans-membrane form that signals via the JAK/STAT pathway, and shorter forms of receptor ${ }^{(4)}$. DC were derived from blood monocytes and effects of probiotic bacteria on gene expression were examined for different forms of receptor. DC expressed both long and short forms of leptin receptor but levels of expression varied in DC from different individuals. Probiotic bacteria reduced relative expression of the long signalling form of receptor (Figure). Parallel studies of formation of intracellular cytokine production showed increases in IL-10 and additional effects of probiotic bacteria on fat uptake into lipid bodies in DC.

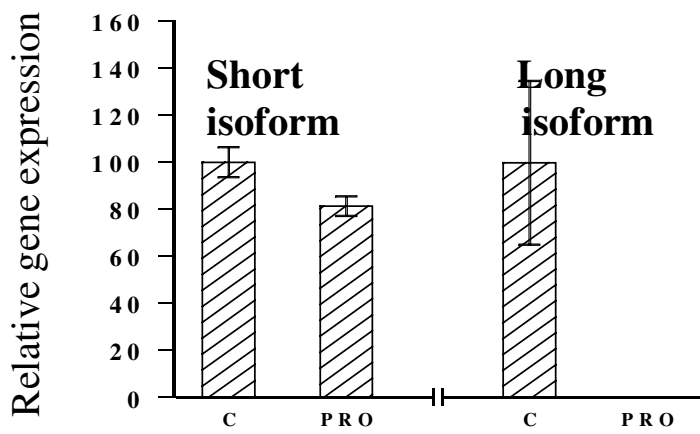

Figure. Dendritic cells were grown from peripheral blood CD14 + monocytes for $7 \mathrm{~d}$ with granulocyte-macrophage colony-stimulating factor and IL-4. Over the last $2 \mathrm{~d}$ some were exposed to a mixture of two probiotic bifidobacteria (B. longum and B. breve). Gene expression of the long and short forms of leptin receptor was measured by PCR in relation to the housekeeping gene ubcH5B. DC expressed both long and short forms of receptor. The probiotic bacteria (PRO) caused preferential down-regulation of the long signalling form of receptor. Values are means with their standard errors represented by vertical bars.

In conclusion, probiotic bacteria caused multiple changes in DC promoting anti-inflammatory effects; selected probiotic bacteria might prove a beneficial therapy for IBD via modulating DC.

1. Bedford PA, TodorovicV, Westcott EDA, Windsor ACJ, English NR, Al-Hassi HO, Raju KS, Mills S \& Knight SC (2006) J Leukoc Biol 80, 546-554.

2. Westcott E, Windsor A, Mattacks C, Pond C \& Knight S (2005) Inflamm Bowel Dis 11, 820-827.

3. Hart AL, Lammers K, Brigidi P, Vitali B, Rizzello F, Gionchetti P, Campieri M, Kamm MA, Knight SC \& Stagg AJ (2004) Gut 53, $1602-1609$.

4. Quinton ND, Laird SM, Tuckerman EM, Cork BA, Li TC \& Blakemore AIF (2003) Am J Reprod Immunol 50, $224-231$. 